\title{
Modelling coevolution in ecological networks with adaptive dynamics
}

\author{
Cang Hui ${ }^{1,2}$ (D) | Henintsoa O. Minoarivelo ${ }^{1,3} \mid$ Pietro Landi $^{1}$
}

${ }^{1}$ Centre for Invasion Biology, Department
of Mathematical Sciences, Stellenbosch
University, Stellenbosch 7602, South
Africa
${ }^{2}$ Mathematical Biosciences Group, African
Institute for Mathematical Sciences, Cape
Town 7945, South Africa
${ }^{3}$ Centre of Excellence in Mathematical
and Statistical Sciences, Wits University,
Johannesburg, Gauteng 2050, South Africa

Correspondence

Cang Hui, Centre for Invasion Biology,

Department of Mathematical Sciences,

Stellenbosch University, Matieland,

Stellenbosch 7602, South Africa.

Email: chui@sun.ac.za

\section{Funding information}

National Research Foundation, Grant/

Award Number: 109244 and 89967; DST-

NRF Centre of Excellence in Mathematical and Statistical Sciences, Grant/Award

Number: BA2017/136

MOS Classification: 92D40; 47J35; 91A22

Communicator: R. Anguelov
Coevolution can impose density-dependent selection through reciprocal biotic interactions on the fitness of involved species, driving directional and disruptive trait evolution and rich evolutionary possibilities. Coevolution has since Darwin been considered a potential path leading to adaptive diversification that could explain the emergence of ecological networks of biotic interactions that harbour multiple interacting species (eg, pollination networks and food webs). Here, we present adaptive dynamics, a powerful tool of evolutionary invasion analysis that explores how quantitative traits undergo incremental evolution, to exploring the emergence of multi-species networks through coevolution. Specifically, we exemplify the feasibility of using adaptive dynamics to investigate trait evolution in 4 ecological networks, driven, respectively, by resource competition, trophic interactions, as well as bipartite mutualistic and antagonistic interactions. We use a set of ordinary differential equations to describe, at different paces, the population dynamics and trait dynamics of involved species assemblages. Through computing ecological equilibrium, invasion fitness, selection gradient and evolutionary singularity, and testing for evolutionary stability and the coexistence criterion of mutual invasibility, we illustrate the typical evolutionary dynamics and the criteria of evolutionary stability and branching in these ecological networks. Results highlight the importance of the form of trait-mediated interaction kernel (ie, interaction strength as a function of trait difference) to adaptive diversification in these coevolutionary systems. We conclude by advocating that biotic interactions between two species can indeed lead to diffuse and even escape-and-radiate coevolution, making the emerged ecological networks an ideal model for studying complex adaptive systems.

\section{KEYWORDS}

adaptive dynamics, complex adaptive networks, ecological networks, evolutionary branching, evolutionary invasion analysis, evolutionary stability

\section{1 | INTRODUCTION}

Evolutionary adaptation has been traditionally viewed as a frequency- or density-independent hill-climbing process in a static fitness landscape, with species often sitting at suboptimal fitness peaks. ${ }^{1}$ The typical dilemma then centres on how a species can journey from one suboptimal fitness peak, via passing through fitness valleys and ditches, to the maximum peak in the entire fitness landscape. The potential diversification from such hill-climbing adaptation has been argued to 
often occur allopatrically along an environmental gradient or through the restriction of gene flows by geographical barriers. ${ }^{2}$ Coevolution, in contrast, often triggers density-dependent selection, where the evolutionary change in one species can lead to a reciprocal change in another species or different phenotypes within the same species to balance their fitness. ${ }^{3,4}$ This nature of evolutionary gaming between involved species pose a dynamic fitness landscape that allows species to dance and trample over the fitness landscape, converging and diverging through directional and disruptive selection, resulting in an eventual "fitness carpet" and a wide variety of evolutionary possibilities. ${ }^{5}$

Coevolution was first conjectured by Darwin ${ }^{6}$ to infer the existence of the sphinx moth, Xanthopan morganii praedicta, a pollinator moth later discovered in 1903 with a 30-cm long proboscis that pollinates the Madagascan orchid, Angraecum sesquipedale. Other similar examples include the coevolution between the long-proboscid fly, Moegistorhynchus longirostris, and the long-tubed iris, Lapeirousia anceps, in the southern Cape (Figure 1). As Darwin ${ }^{6}$ put it, the two coevolving species "might slowly become, either simultaneously or one after the other, modified and adapted in the most perfect manner, by the continued preservation of individuals presenting mutual and slightly favourable deviation of structure." Recent phylogenetic evidence supports coevolution being a potential source of clade diversification. For instance, the mutualistic interaction of seed dispersal by ants could have promoted diversification in flowering plants. ${ }^{7}$ The pollination of flowers by insects could explain the rich diversity of angiosperms (flowering plants) over gymnosperms. Escape-and-radiate coevolution between plants and herbivores are also common, such as between the leaf beetles Blepharida and their host trees Bursera ${ }^{8}$ and between endosymbiotic bacteria Buchnera aphidicola and aphids. ${ }^{9}$ All these clues have suggested that coevolution can potentially lead to rich evolutionary trajectories via density-dependent selection and, especially, the possibility of diversification and polymorphism via evolutionary branching from disruptive selection in the system. This suggests the potential of network emergence through adaptive diversification, ${ }^{10}$ adding support to the hypothesis of Ehrlich and Raven ${ }^{11}$ on the coevolutionary origin of biotic interactions in ecological networks.

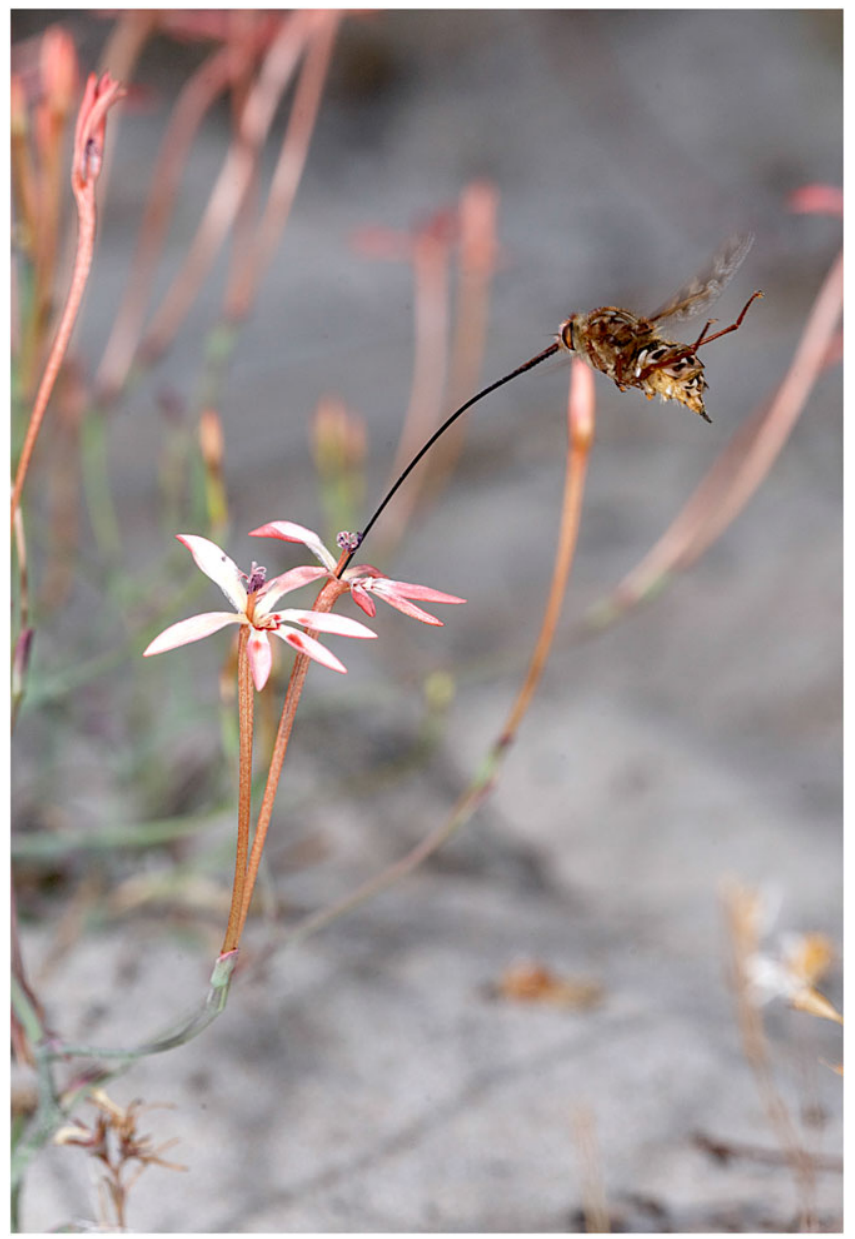

FIGURE 1 The coevolution between the long-proboscid fly, Moegistorhynchus longirostris, and the long-tubed iris, Lapeirousia anceps, in the Western Cape of South Africa. Photo credit: Anton Pauw [Colour figure can be viewed at wileyonlinelibrary.com] 
Several studies have explored how coevolution can affect the dynamics and structures of ecological networks of biotic interactions. In particular, Rezende et $\mathrm{al}^{12}$ examined the evolution of traits along phylogenetic trees, which partially explained the nested architecture in mutualistic networks. They found that in 50\% of the empirical networks examined, phylogenetic proximity between paired species was positively correlated with their interaction similarities, suggesting the tendency for closely related species to interact with the same partners. ${ }^{13}$ This means that observed network architecture could partially reflect the distinct coevolutionary history of involved lineages (eg, phylogenetic asymmetry and accelerated evolution). Some studies have further implemented the coevolutionary effect of reciprocal selection on involved taxa. For example, Guimarães et $\mathrm{al}^{14}$ modelled the coevolution of traits in mutualistic networks and found a higher convergence of traits in super-generalist species that play important roles in maintaining network organisation and stability. Nuismer et $\mathrm{al}^{15}$ showed that coevolutionary selection could increase network connectance while altering the pattern of nestedness. Minoarivelo et $\mathrm{al}^{16}$ designed a model describing the evolution of pairwise interactions as Markov processes and managed to produce network architectures, including node-degree distributions, resembling empirical networks.

The role of trait-mediated biotic interactions in triggering density-dependent selection has been highlighted in recent literature, with many theoretical studies attempting to elucidate how these trait-mediated interactions in coevolutionary networks could trigger disruptive selection and adaptive diversification. ${ }^{17}$ In particular, 2 coevolving species are engaging with an evolutionary arms race through the interaction of their functional traits that affect each other's fitness. Such specific coevolution can typically lead to matched traits through convergence evolution in mutualistic systems and evolutionary cycles, known as the Red Queen dynamics, in antagonistic systems. The coevolution between 2 species could lead to diffusive and then escape-and-radiate coevolution, where multiple species from a functional guild affect each other's fitness by their own evolutionary changes, driving adaptive diversification from repeatedly occurring disruptive selection in the system. ${ }^{18}$ Such emergence of ecological networks through adaptive diversification from coevolution is the concern here. In the following, we use a powerful tool of evolutionary invasion analysis, known as adaptive dynamics, ${ }^{19-21}$ to explore the patterns and conditions of adaptive diversification and evolutionary branching in ecological networks of resource competition, mutualism, antagonism, and food webs. Specifically, we explore the feasibility of using adaptive dynamics to explore trait evolution in ecological networks by specifying under what conditions a pair of interacting species can potentially trigger disruptive selection and diversify through specific, diffuse, and even escapeand-radiate coevolution. Note, we do not consider coevolution in spatial networks, where nodes represent geographical patches connected by gene flows. Considering spatial networks raises some complex but distinct questions, as mutants could outcompete residents locally in some patches and then spill over into other patches via migration. ${ }^{22-24}$

\section{ADAPTIVE DYNAMICS}

Evolutionary trajectory can be portrayed as the optimisation of the life-history strategies (or loosely defined as phenotypical traits) through feasible pathways towards the fitness maximum, if any, in the phenotype-dependent fitness landscape of a species. This definition relies on 2 premises. First, within the attainable trait set that is normally bounded by the physiological limit, there exists a trait that has the maximum fitness in the fitness landscape. This trait is called the evolutionarily stable strategy (ESS), as no other traits can competitively replace it. Second, this trait with the maximum fitness can be reached through incremental evolutionary changes from the current standing trait; that is, this maximum-fitness trait needs to be convergence (asymptotically) stable. A convergence stable ESS is named a continuously stable strategy (CSS). Evolution concerns essentially the trait dynamics in the fitness landscape, towards a CSS. ${ }^{25}$ Evolutionary invasion analysis is a set of quantitative techniques designed to address these 2 premises: the existence of an ESS and the incremental evolution through the invasion of a rare mutant trait into a resident population. ${ }^{26,27}$ Notably, the invading trait is normally considered not far from the resident one (ie, incremental evolution); that is, we are looking for a local CSS, strategies that are convergence stable and cannot be invaded by slightly different traits. However, with the onslaught of global environmental changes, many nonindigenous species or genotypes are constantly being introduced into resident ecosystems, suggesting the increasing relevance of searching for the global CSS in an evolutionary system. ${ }^{28,29}$

Developed by game theoreticians, ${ }^{30}$ population geneticists ${ }^{31}$ and theoretical ecologists, ${ }^{32}$ adaptive dynamics (AD hereafter) is a powerful analytical tool for studying the evolution of quantitative traits or phenotypic characters. ${ }^{19}$ It studies evolutionary changes induced by rare and small mutations when fitness is density- or frequency-dependent. ${ }^{33}$ Because individuals from different resident species can interact within each other in a local community, their fitness depends not only on their own traits (strategies) but also the frequency or density of individuals with other different traits, a typical issue of game theory. The evolution of traits can be evaluated by examining the invasion and survival 
of rare mutants in a community dominated by resident populations at their stable equilibriums. To this end, the canonical equation of $\mathrm{AD}$ has been used to describe the evolution of traits under directional selection through the continuous invasion of rare mutants into resident populations. ${ }^{21}$ The most interesting feature of AD is its capacity to formally describe the condition of evolutionary branching ${ }^{20,34,35}$ : At an evolutionarily singular strategy, where directional selection ceases (ie, equilibrium of the canonical equation where the selection gradient vanishes), the fitness landscape can be found to locate at either its maxima or minima, determined by the second-order derivatives of the mutant fitness at the singular strategy. In the latter case, if the resident and the initially resembling mutant can further competitively coexist (known as protected dimorphism), the disruptive selection posed by the fitness minima could then give rise to an evolutionary branching, a typical phenomenon of adaptive diversification.

\section{3 | RESOURCE COMPETITION}

Resource competition is the most common biotic interaction during community assembly. We thus first illustrate the standard procedure of using AD in a resource-competition model. For a given set of $n$ traits, changes in population densities $u_{i}(i=1,2, \ldots, n)$ are described by the Lotka-Volterra model,

$$
\frac{d u_{i}}{d t}=r u_{i}\left(1-\frac{\sum_{l} \alpha\left(x_{i}, x_{l}\right) u_{l}}{k\left(x_{i}\right)}\right)
$$

where $r$ is the intrinsic population growth rate, $k\left(x_{i}\right)$ the trait-dependent carrying capacity, and $\alpha\left(x_{i}, x_{l}\right)$ is the competition strength between individuals with trait value $x_{i}$ and $x_{l}$. Because mutations only occur at a low rate, the population densities are considered to be already at their equilibriums when a mutation happens. In this regard, we need to distinguish 2 different time scales in the concept of AD: a slow evolutionary time scale (including the slow trait shifted by directional selection and the even slower evolutionary branching by disruptive selection) and a fast ecological time scale of population dynamics. Let $x^{\prime}$ be the trait value of a rare mutant, $X=\left(x_{1}, x_{2}, \ldots, x_{l}, \ldots, x_{n}\right)$ the resident traits, and $u_{i}^{*}$ the population density at equilibrium. The invasion fitness of the mutant can be described as its per capita growth rate when setting its initial density to be negligible:

$$
f\left(X, x^{\prime}\right)=r\left(1-\frac{\sum_{l=1}^{n} \alpha\left(x^{\prime}, x_{l}\right) u_{l}^{*}}{k\left(x^{\prime}\right)}\right) .
$$

The selection gradient of population $i$,

$$
g_{i}\left(x_{i}\right)=\left.\frac{\partial f\left(X, x^{\prime}\right)}{\partial x^{\prime}}\right|_{x^{\prime}=x_{i}}=-r \frac{k\left(x_{i}\right) \sum_{l=1}^{n} \partial_{x^{\prime}}\left(\alpha\left(x_{i}, x_{l}\right) u_{l}^{*}\right)-\partial_{x^{\prime}}\left(k\left(x_{i}\right)\right)\left(\sum_{l=1}^{n} \alpha\left(x_{i}, x_{l}\right) u_{l}^{*}\right)}{k\left(x_{i}\right)^{2}},
$$

determines the speed of directional selection. Assuming Gaussian forms (scaled normal such that the maximum value equals 1) of resource and competition kernels, ${ }^{17,36}$

$$
\begin{aligned}
& k(x)=K \cdot N\left(x^{\max }, \sigma_{k}, x\right), \\
& \alpha\left(x^{\prime}, x\right)=N\left(0, \sigma_{\alpha}, x^{\prime}-x\right),
\end{aligned}
$$

where $K$ is the maximum carrying capacity occurring at trait $x^{\max }$, while $\sigma_{k}$ and $\sigma_{\alpha}$ represent the standard deviation (width) of the resource and competition kernels, respectively. We have the following selection gradient for the monomorphic case $u_{1}^{*}=k\left(x_{1}\right)$ :

$$
g\left(x_{1}\right)=-r \frac{x_{1}-x^{\max }}{\sigma_{\mathrm{k}}^{2}} .
$$

The evolutionary dynamics of trait $x_{i}$ can be depicted by the canonical equation as being proportional to the selection gradient, ${ }^{19}$ 


$$
\dot{x}_{i}=\varepsilon \cdot u_{i}^{*} g_{i}\left(x_{i}\right)
$$

where $\varepsilon$ is a parameter related to the rate and variation of mutation. If the directional selection pushes the traits to become unfeasible (ie, the population density at equilibrium becomes equal to or less than zero), we are witnessing an evolutionary extinction (suicide, ${ }^{37}$ runaway, or murder $^{21}$ ).

Let $X^{*}=\left(x_{1}^{*}, x_{2}^{*}, \ldots, x_{l}^{*}, \ldots, x_{n}^{*}\right)$ indicate the trait vector where the selection gradients of all resident traits disappear, termed an evolutionary singularity (in the above example, $x_{1}^{*}=x^{\max }$ ). The singularity is convergence stable if all eigenvalues of the Jacobian of the canonical equations have negative real parts ${ }^{19,21}$; in this case $(n=1)$,

$$
\left.\frac{d\left(\varepsilon \cdot u_{i}^{*} g\left(x_{1}\right)\right)}{d x_{1}}\right|_{x_{1}=x_{1}^{*}}=\frac{-r \varepsilon \cdot k\left(x_{1}^{*}\right)}{\sigma_{k}^{2}}<0 .
$$

The singularity $x_{i}^{*}$ represents a fitness minimum, an indication of disruptive selection, if the curvature of fitness landscape at the trait $x_{i}^{*}$ is greater than zero,

$$
\begin{gathered}
\left.\frac{\partial^{2} f\left(X, x^{\prime}\right)}{\partial x^{\prime 2}}\right|_{x^{\prime}=x_{i}^{*}}>0 \\
X=X^{*}
\end{gathered}
$$

which allows traits other than the singularity to invade ${ }^{20,34,35}$; intuitively, the curvature is also a measure of the strength of disruptive selection. To have an evolutionary branching, not only the singularity needs to be a fitness minimum and under disruptive selection but also the 2 morphs emerged from the evolutionary branching need to be protected ${ }^{20}$; that is, the two morphs $\left(x^{\prime}\right.$ and $\left.x^{\prime \prime}\right)$ can coexist and invade each other:

$$
\left.\left(\frac{\partial^{2} f\left(x^{\prime}, x^{\prime \prime}\right)}{\partial x^{\prime 2}}+\frac{\partial^{2} f\left(x^{\prime}, x^{\prime \prime}\right)}{\partial x^{\prime \prime}}\right)\right|_{x^{\prime}=x^{\prime \prime}=x_{i}^{*}}>0
$$

In a monomorphic population $(n=1)$, the convergence stability and the evolutionary instability of the singular strategy automatically imply that inequality 9 is satisfied and, therefore, dimorphism is protected.

If the singularity represents a fitness minimum and convergence stable but the dimorphism cannot be protected, it is called an evolutionary trap ${ }^{38}$ or a terminal point. ${ }^{21}$ In that case, either the mutant or the resident population, not both, would persist and replace the other population. In our example, the evolutionary branching at the monomorphic singular strategy requires the resource kernel being wider than the competition kernel $\left(\sigma_{k}>\sigma_{\alpha}\right)$, meaning that, through the evolutionary branching, the loss in resource acquisition must be smaller than the advantage of reducing competition.

FIGURE 2 Trait diversification under resource competition. Lines represent trait dynamics along the evolutionary time, starting from a monomorphic population and gradually diversifying into 5 different morphs. Background colour represents the invasion fitness. Parameter values: $r=K=1, x^{\max }=0, \sigma_{k}=1.25$, and $\sigma_{\alpha}=1$ [Colour figure can be viewed at wileyonlinelibrary.com]

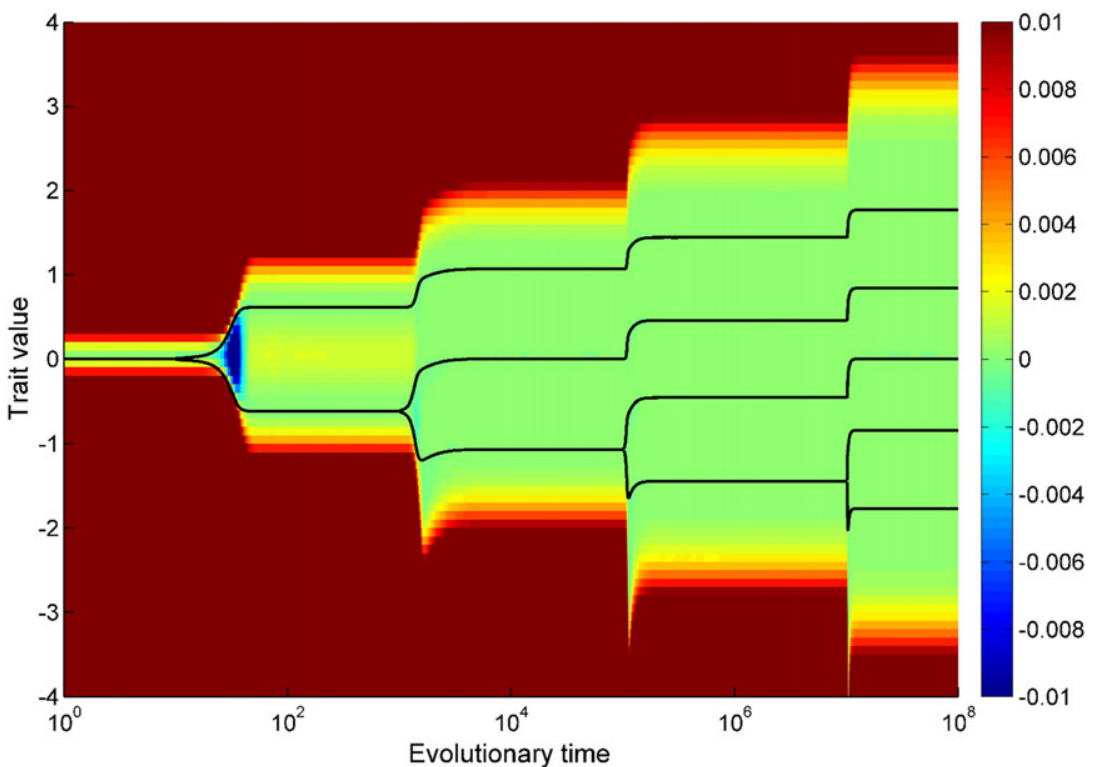


Such a condition has been shown to also hold for the dimorphic singular strategy. ${ }^{39}$ Therefore, a monomorphic population as depicted by Equation 1, with its trait dynamics depicted by Equation 6, would potentially undergo subsequent evolutionary branching events leading to polymorphism (Figure 2). Moreover, our simulation in Figure 2 and Dercole et $\mathrm{al}^{40}$ support the idea that the monomorphic branching condition in fact can be generalised for systems with $n>2$, although successive branching events become weaker and slower (notice the logarithmic scale on the time axis and the decreasing curvature of the fitness landscape in Figure 2).

\section{4 | MUTUALISTIC COEVOLUTION}

Species maintain a mutualistic interaction by providing each other with benefits (fitness gains). For instance, the specialisation of the interaction between the yucca moth (Tegeticula synthetica) and the Joshua tree (Yucca brevifolia) has been attributed to their specific coevolution..$^{41}$ While the seed of Joshua tree is the only food source for the yucca moth, the yucca moth is the only pollinator of the Joshua tree. By changing the interacting functional traits through evolution (eg, the proboscis of pollinators and the floral tube of flowers; see Figure 1), mutualistic interactions can lead to intriguing coevolutionary games. Some long-standing mutualistic interactions can further lead to the symbioses of both partners such as between legumes and rhizobia, with rhizobia fixing atmospheric nitrogen within root nodules that legumes can use, while simultaneously receiving carbon resources from the plant. ${ }^{42}$ However, in a large network that is dominated by mutualistic interactions, species are simultaneously under different and often conflicting selection pressures (such as intraspecific competition or escape from predation). Moreover, maintaining a symbiotic or mutualistic relationship can be costly. As such, a major challenge has been to unveil the protective mechanism that the involved partners have adopted for discerning and correcting the cheating behaviour that can be disastrous to the functioning of the system. ${ }^{43}$ To this end, although a number of empirical studies have emphasised the important role of mutualistic interactions in fostering multispecies coexistence, ${ }^{44,45}$ mutualistic coevolution might not be the main driver of adaptive diversification in mutualistic networks. ${ }^{46}$

To model the coevolution of mutualistically interacting species in a community and explore the importance of mutualistic interactions in adaptive diversification, we use an $\mathrm{AD}$ model based on the Lotka-Volterra model of mutualism with a Holling type II functional response (see also Ferrière et $\mathrm{al}^{47}$ and Dercole ${ }^{48}$ where mutualism is instead obligate). The population dynamics is governed by the demography, including intrinsic population growth and density dependence, and the additional contribution from the mutualistic interaction. Let there be $n$ functional morphs of animals and $m$ functional morphs of plants. Each functional morph, indexed by $i$ for animals and $j$ for plants, is characterised by its population density $u_{i}$ and $v_{j}$, respectively. In a pollination system, the functional trait could, for example, be related to its feeding apparatus such as the length of the proboscis of the pollinator. The functional trait for the plant could be related to the flower's morphology, such as the length of its floral tube. We denote the trait of animal morph $i$ by $x_{i}$ and the trait of plant morph $j$ by $y_{j}$. The population dynamics of the AD system is thus given by

$$
\begin{aligned}
& \frac{d u_{i}}{d t}=r^{\prime} u_{i}\left(1-\frac{\sum_{l=1}^{n} \alpha_{i l}^{\prime}\left(x_{i}, x_{l}\right) u_{l}}{k_{A}\left(x_{i}\right)}\right)+\frac{u_{i} c \sum_{l=1}^{m} \gamma_{i l}\left(x_{i}, y_{l}\right) v_{l}}{1+h \sum_{l=1}^{m} \gamma_{i l}\left(x_{i}, y_{l}\right) v_{l}}, \\
& \frac{d v_{j}}{d t}=r^{\prime \prime} v_{j}\left(1-\frac{\sum_{l=1}^{m} \alpha_{j l}^{\prime \prime}\left(y_{j}, y_{l}\right) v_{l}}{k_{P}\left(y_{j}\right)}\right)+\frac{v_{j} c \sum_{l=1}^{n} \gamma_{l j}\left(x_{l}, y_{j}\right) u_{l}}{1+h \sum_{l=1}^{n} \gamma_{l j}\left(x_{l}, y_{j}\right) u_{l}},
\end{aligned}
$$

where $r^{\prime}$ and $r^{\prime \prime}$ are the intrinsic growth rates of animal and plant, respectively; $h$ is the handling time; and $c$ is a parameter controlling the magnitude of mutualistic benefit. Specifically, we assigned the trait-dependent carrying capacity to be a quadratic function of trait value ${ }^{39}$ :

$$
k_{A}\left(x_{i}\right)=K_{A}\left(1-\left(\frac{x_{i}-x^{\mathrm{max}}}{\delta_{A}}\right)^{2}\right),
$$

within a certain range of viable trait (when $\left|x_{i}-x^{\max }\right| \leq \delta_{A}$ ) and $k_{A}\left(x_{i}\right)=0$ if outside the viable trait range. $K_{A}$ is the carrying capacity for optimal trait $x^{\max }$, and $\delta_{A}$ represents the resource niche width accessible to the animals. The carrying 
capacity for plants, $k_{P}\left(y_{j}\right)$, can be similarly defined. The intratrophic competition kernels $\left(\alpha^{\prime}\right.$ and $\left.\alpha^{\prime \prime}\right)$ are set to let more similar morphs suffer stronger competition and are assumed to follow a Gaussian function, scaled normal with a maximum value ${ }^{17,46,49}$ equal to 1 :

$$
\begin{aligned}
& \alpha_{i l}^{\prime}\left(x_{i}, x_{l}\right)=N\left(0, \sigma_{A}, x_{i}-x_{l}\right), \\
& \alpha_{j l}^{\prime \prime}\left(y_{j}, y_{l}\right)=N\left(0, \sigma_{P}, y_{j}-y_{l}\right),
\end{aligned}
$$

where the standard deviations $\left(\sigma_{A}\right.$ and $\left.\sigma_{P}\right)$ describe the width of the competition kernel for animal and plant, respectively. The cross-trophic mutualistic strength,

$$
\gamma_{i j}\left(x_{i}, y_{j}\right)=N\left(0, \sigma_{m}, x_{i}-y_{j}\right)
$$

reflects the assumption that matching traits between animal and plant bring high profit to each other. The parameter $\sigma_{m}$ controls the tolerance level of successful interactions to the trait difference of involved traits. ${ }^{15}$ Considering a monomorphic resident animal population with trait $x$ coexisting with a monomorphic plant population with trait $y$ at equilibrium abundances $\left(u^{*}\right.$ and $\left.v^{*}\right)$, the invasion fitness for a mutant animal with trait $x$ and for a mutant plant with trait $y^{\prime}$ can be given by

$$
\begin{aligned}
& f_{A}\left(x, x^{\prime}\right)=r^{\prime}\left(1-\frac{\alpha^{\prime}\left(x^{\prime}, x\right) u^{*}}{k_{A}\left(x^{\prime}\right)}\right)+\frac{c \gamma\left(x^{\prime}, y\right) v^{*}}{1+h \gamma\left(x^{\prime}, y\right) v^{*}}, \\
& f_{P}\left(y, y^{\prime}\right)=r^{\prime \prime}\left(1-\frac{\alpha^{\prime \prime}\left(y^{\prime}, y\right) v^{*}}{k_{P}\left(y^{\prime}\right)}\right)+\frac{c \gamma\left(x, y^{\prime}\right) u^{*}}{1+h \gamma\left(x, y^{\prime}\right) u^{*}},
\end{aligned}
$$

and the selection gradients on animal and plant traits by the following:
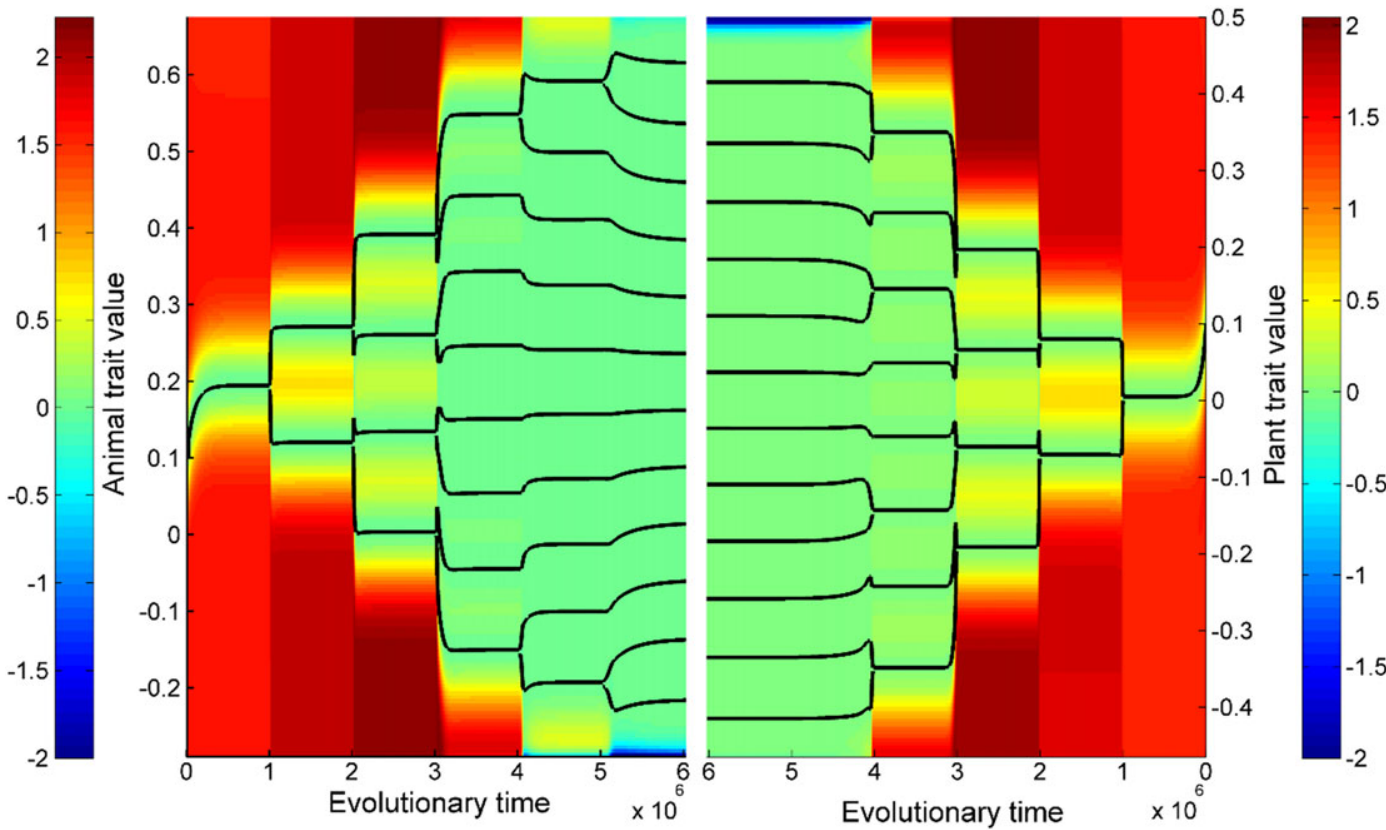

FIGURE 3 Trait diversification under mutualistic coevolution. Lines represent trait dynamics along the evolutionary time, starting from a monomorphic population and gradually diversifying into 12 different morphs on each side of the trophic. Background colour represents the invasion fitness. Parameter values: $r^{\prime}=r^{\prime \prime}=1, K_{A}=0.5, K_{P}=1, x^{\max }=0.2, y^{\max }=0, \delta_{A}=\delta_{P}=0.5, \sigma_{A}=\sigma_{P}=0.05, \sigma_{m}=1, c=0.75$, and $h=0.5$ [Colour figure can be viewed at wileyonlinelibrary.com] 


$$
\begin{aligned}
& g_{A}(x)=-r^{\prime} \frac{k_{1}(x) u^{*} \partial_{x^{\prime}} \alpha^{\prime}(x, x)-\alpha^{\prime}(x, x) u^{*} \partial_{x^{\prime}} k_{1}(x)}{k_{A}(x)^{2}}+\frac{c v^{*} \partial_{x^{\prime}} \gamma(x, y)}{\left(1+h \gamma(x, y) v^{*}\right)^{2}}, \\
& g_{P}(y)=-r^{\prime \prime} \frac{k_{2}(y) v^{*} \partial_{y^{\prime}} \alpha^{\prime \prime}(y, y)-\alpha^{\prime \prime}(y, y) v^{*} \partial_{y^{\prime}} k_{2}(y)}{k_{P}(y)^{2}}+\frac{c u^{*} \partial_{y^{\prime}} \gamma(x, y)}{\left(1+h \gamma(x, y) u^{*}\right)^{2}} .
\end{aligned}
$$

The population dynamics together with the canonical equations of AD of the 2 traits were numerically solved with initially a pair of monomorphic populations and a unit initial density for both plants and animals. To take into account the time-scale separation between ecological and evolutionary dynamics, we set the mutation rate to a small constant $\left(\varepsilon=10^{-3}\right)$. The 3 conditions for an evolutionary branching were examined once the system had reached its singularity.

As illustrated in Figure 3, mutualistic interactions between a monomorphic animal population and a monomorphic plant population, plus resource competition (Equation 10), can trigger disruptive selection and lead to diffuse and even escape-and-radiate coevolution. Evolutionary branching was more likely to happen for stronger tolerance to trait difference (larger $\sigma_{m}$ ) and narrower competition kernels (smaller $\sigma_{A}$ and $\sigma_{P}$ ). ${ }^{5}$ Narrow competition kernels suggest an intense trait-specific competition, ie, strong negative frequency dependence, which has been argued a common condition for adaptive diversification. ${ }^{10,50}$ A strong tolerance to trait difference, as in many generalist species, ensures that benefit gained from mutualism can provide sufficient resource to sustain the survival of new mutants. Hence, even in a community dominated by mutualistic interactions, intratrophic resource competition is still the main driver of adaptive diversification.

\section{5 | ANTAGONISTIC COEVOLUTION}

Antagonistic interactions often occur through the mediation between the foraging traits of predators and the antiforaging traits of their prey, such as between the speed of cheetahs and the agility of gazelles,${ }^{51}$ between the fish stock and fishery policies, ${ }^{52}$ and between the toxicity of rough skinned newts (Taricha granulosa) and the resistance of garter snakes (Thamnophis sirtalis). ${ }^{53}$ The drastic antagonistic warfare between plants and herbivores has resulted in the syntheses of diverse secondary compounds by plants as a defence mechanism against herbivores. ${ }^{54}$ Coevolution via antagonistic interactions can lead to rapid radiation of defensive traits ${ }^{55}$ and interesting phenomena of aposematism and mimicry. ${ }^{56}$ Antagonism is also typical between the arms race of hosts and their parasites or pathogens. Reed warblers distinguish artificial eggs closely resembling their own, while brood-parasitic cuckoos Cuculus canorus produce eggs that are increasingly difficult for host warblers to recognise. ${ }^{57}$ Examples of host-parasite coevolution abound in many infectious diseases. Planktonic crustacean Daphnia magna can control the infectivity of the parasitic bacterium Pasteuria ramose while facing an ever-increasing virulence of the parasite. ${ }^{58}$ Of course, the coevolution between host and pathogens does not necessarily lead to the escalation of virulence as many pathogens require the wellbeing of their hosts for vertical transmission. The weakening virulence of human immunodeficiency virus can be considered an example of reduced virulence from the antagonistic coevolution between the virulence and the host's immunity. ${ }^{59}$ Again, the key to elucidating an antagonistic interaction is to identify the interacting traits that affect the predator's energy intake and the prey's survival.

Many laboratory experiments have been conducted for observing the effect of antagonistic interactions on the diversification in coevolutionary systems. Specifically, the coevolution between hosts and their respective parasites has been extensively studied. Results suggest that, although hosts often develop resistances against their parasites, this often triggers the adaptive diversification in the parasites, which in turn diversifies the resistance strategies of hosts. ${ }^{60}$ Adaptive diversification in the prey, resulted from counter-adaptation against predation, can further trigger the subsequent diversification of the predator's foraging traits. ${ }^{51}$ This escalation in coadaptation between traits of the predator and the prey, commonly termed as the arms race dynamics, is common in antagonistic systems. In what follows, we once again make use of the Lotka-Volterra model for depicting the dynamics of $n$ predator densities $\left(u_{i}\right)$ and $m$ prey densities $\left(v_{i}\right)$, specifically adapted for host-parasite interactions (we do not consider here hostspecific parasitism): 


$$
\begin{aligned}
& \frac{d u_{i}}{d t}=-r^{\prime} u_{i}\left(1+\frac{\sum_{l=1}^{n} \alpha_{i l}^{\prime}\left(x_{i}, x_{l}\right) u_{l}}{k_{P}\left(x_{i}\right)}\right)+\frac{\lambda u_{i} \sum_{l=1}^{m} a \gamma_{i l}\left(x_{i}, y_{l}\right) v_{l}}{1+h \sum_{l=1}^{m} a \gamma_{i l}\left(x_{i}, y_{l}\right) v_{l}}, \\
& \frac{d v_{j}}{d t}=r^{\prime \prime} v_{j}\left(1-\frac{\sum_{l=1}^{m} \alpha_{j l}^{\prime \prime}\left(y_{j}, y_{l}\right) v_{l}}{k_{H}\left(y_{j}\right)}\right)-\sum_{l=1}^{n} \frac{v_{j} a \gamma_{l j}\left(x_{l}, y_{j}\right) u_{l}}{1+h \sum_{k=1}^{m} a \gamma_{l k}\left(x_{l}, y_{k}\right) v_{k}}
\end{aligned}
$$

where functions for intratrophic competition are similar to those in the mutualistic model (Equation 12) with standard deviations $\sigma_{P}$ and $\sigma_{H}$ for the parasite and the host, respectively. The carrying capacities, $k_{P}\left(x_{i}\right)$ and $k_{H}\left(y_{j}\right)$, are assumed to follow Gaussian functions of the traits:

$$
\begin{aligned}
& k_{P}\left(x_{i}\right)=K_{P} N\left(x^{\max }, \delta_{P}, x_{i}\right), \\
& k_{H}\left(y_{j}\right)=K_{H} N\left(y^{\max }, \delta_{H}, y_{j}\right) .
\end{aligned}
$$

The attack rate of the host $j$ with trait $y_{j}$ by the parasite $i$ with trait $x_{i}$ is governed by a Gaussian function of trait difference,

$$
\gamma_{i j}\left(x_{i}, y_{j}\right)=N\left(\mu_{p}, \sigma_{p r}, x_{i}-y_{j}\right) .
$$

The attack rate becomes maximal when the host trait value $\left(y_{j}\right)$ is the parasite trait value $\left(x_{i}\right)$ minus $\mu_{\mathrm{p}}$, which defines the optimal difference between traits of parasites and their hosts so that the parasites can most efficiently attack/infect such hosts. Parameter $\lambda$ represents the conversion rate, while parameter $a$ scales the attack rate. Considering a monomorphic resident predator population with trait $x$ coexisting with a monomorphic prey population with trait $y$ at equilibrium abundances $\left(u^{*}\right.$ and $\left.v^{*}\right)$, the invasion fitness for a mutant parasite with trait $x^{\prime}$ and for a mutant host with trait $y^{\prime}$ are given by

$$
\begin{aligned}
& f_{P}\left(x, x^{\prime}\right)=-r^{\prime}\left(1+\frac{\alpha^{\prime}\left(x^{\prime}, x\right) u^{*}}{k_{P}\left(x^{\prime}\right)}\right)+\frac{\lambda a \gamma\left(x^{\prime}, y\right) v^{*}}{1+h a \gamma\left(x^{\prime}, y\right) v^{*}}, \\
& f_{H}\left(y, y^{\prime}\right)=r^{\prime \prime}\left(1-\frac{\alpha^{\prime \prime}\left(y^{\prime}, y\right) v^{*}}{k_{H}\left(y^{\prime}\right)}\right)-\frac{a \gamma\left(x, y^{\prime}\right) u^{*}}{1+h a \gamma(x, y) v^{*}},
\end{aligned}
$$

and the selection gradients on predator and prey traits are given by

$$
\begin{aligned}
& g_{P}(x)=-r \frac{k_{P}(x) u^{*} \partial_{x^{\prime}} \alpha^{\prime}(x, x)-\alpha^{\prime}(x, x) u^{*} \partial_{x^{\prime}} k_{P}(x)}{k_{P}(x)^{2}}+\frac{\lambda a v^{*} \partial_{x^{\prime}} \gamma(x, y)}{\left(1+h a \gamma(x, y) v^{*}\right)^{2}}, \\
& g_{H}(y)=-r^{\prime \prime} \frac{k_{H}(y) v^{*} \partial_{y^{\prime}} \alpha^{\prime \prime}(y, y)-\alpha^{\prime \prime}(y, y) v^{*} \partial_{y^{\prime}} k_{H}(y)}{k_{H}(y)^{2}}-\frac{a u^{*} \partial_{y^{\prime}} \gamma(x, y)}{1+h a \gamma(x, y) v^{*}} .
\end{aligned}
$$

As above, the evolutionary dynamics of the traits can be derived from the canonical equation of the AD (see also Landi et $\mathrm{al}^{51}$ and Landi and Dercole ${ }^{61}$ for details).

It is clear that the antagonistic interaction can lead to disruptive selection and evolutionary branching (Figure 4 and Landi et $\mathrm{al}^{51}$ ). For the above model, evolutionary branching is more likely to occur in parasites, especially when the competition kernel of parasites is relatively narrow $\left(\right.$ small $\left.\sigma_{P}\right){ }^{5}$ Diversification in the host is more likely to happen when its competition kernel is narrow (small $\sigma_{H}$ ) and can only happen exclusively in the host if the competition kernel of the parasites is also narrow (small $\sigma_{P}$ ). In other words, the host cannot diversify if the competitive interference between parasites is only weakly trait-dependent (large $\left.\sigma_{P}\right) .^{5}$ Moreover, when the competition kernel of parasites is wide but that of the host is narrow, the system becomes unstable, suggesting that the increased mortality due to intensive intratrophic competition has exceeded the capacity that the cross-trophic energy flow can support, producing a zone of evolutionary suicide. $^{5}$ Another interesting feature of prey-predator coevolution is the Red Queen dynamics, ${ }^{62}$ ie, the convergence of trait evolution to a nonstationary regime (eg, limit cycle, ${ }^{63,64}$ chaotic attractor, ${ }^{65,66}$ or more complex evolutionary scenar$\left.\operatorname{ios}^{4,67,68}\right)$. The name was inspired by a quote from the Red Queen Alice in wonderland: "Now, here, you see, it takes all 

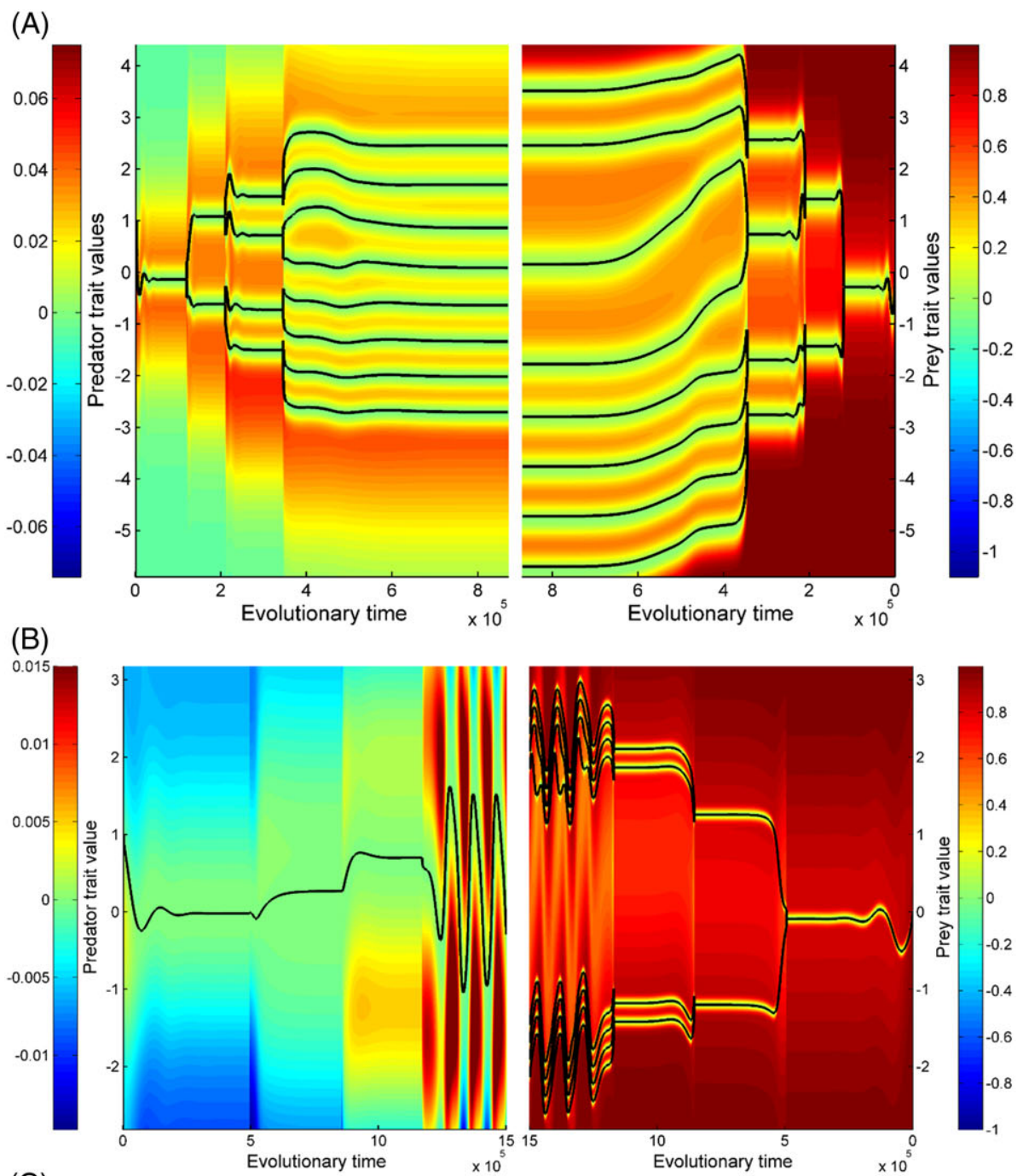

(C)

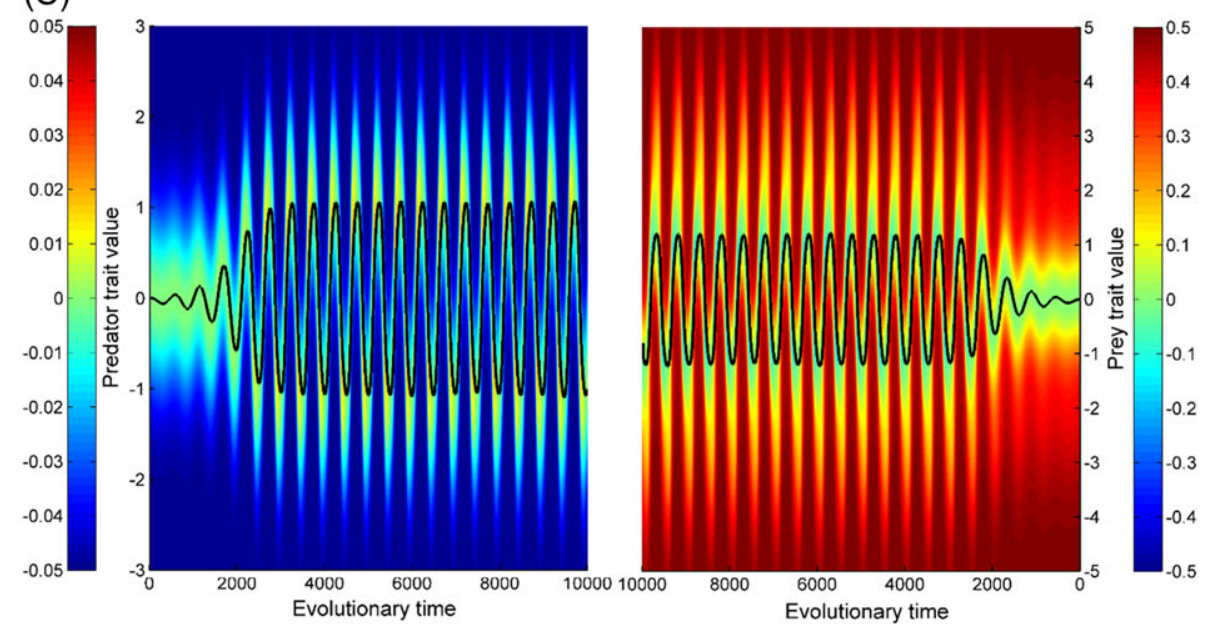

FIGURE 4 Trait evolution under antagonistic coevolution. A, Trait diversification under antagonistic coevolution derived from the model in Equation 16. Red Queen coevolutionary cycle of prey and predator in B, polymorphic (derived from the model in Equation 16) and C, monomorphic populations (derived from the model in Landi et $\mathrm{al}^{51}$ ). Lines represent trait dynamics along the evolutionary time, starting from a monomorphic population. Background colour represents the invasion fitness. Parameter values are the same as in Figure 2 except that in A, $r^{\prime}=0.005, \delta_{P}=\delta_{H}=2.3, \sigma_{P}=\sigma_{H}=0.5, \sigma_{p r}=1, a=2, \mu_{p}=0$, and $\lambda=0.75$; in B, $\sigma_{P}=2, \sigma_{H}=0.05$, and $\sigma_{p r}=1.25$; in $\mathrm{C}$, as in B except $r^{\prime}=0.05, r^{\prime \prime}$ $=0.5, \sigma_{H}=2, a=1$, and $\lambda=0.5$ [Colour figure can be viewed at wileyonlinelibrary.com] 
the running you can do, to keep in the same place.” In biology, it refers to the hypothesis that organisms must constantly adapt not only to gain reproductive advantage but also simply to survive against ever-evolving opposing organisms in an ever-changing environment. The above host-parasite model can exhibit such Red Queen trait dynamics (Figure 4B). Such a never-ending chase of evolutionary traits occurs also in the prey-predator model of Landi et al, ${ }^{51}$ which is slightly different from the model presented above, in that predators do not compete with each other $\left(\alpha^{\prime}=0\right)$, that handling time is trait-dependent, and that the attack rate is a leptokurtic function with an exponent of 1.8 instead of the Gaussian function in the above model with an exponent of 2 (ie, a function with a thin peak describing a more specialised predator), Figure 4C.

\section{I FOOD WEBS}

Food webs exhibit more complex dynamics as they encompass a variety of interactions such as antagonism and competition across multiple trophic levels. For such a complex system, mathematical models of coevolution can provide insights as to the conditions that foster diversification within and cross trophic levels, with the potential for cannibalism. ${ }^{68-71}$ In particular, Brännström et $\mathrm{al}^{18}$ have explored the role of body size as the key functional trait in initiating, structuring, and maintaining food web biodiversity. Here, we use a similar model but without interference competition in the heterotrophs to focus on the conditions that promote diversification in a food web, with specific emphasis on the role of the consumption kernel (explained below). Consider a basal autotrophic resource $(i=0)$ and $n$ heterotrophic morphs with population densities $\left(u_{i}\right)$ such that each morph is associated with its average body size $s_{i}$. While defining the trait value of each morph as the body size relative to the autotroph, $x_{i}=\ln \left(s_{i} / s_{0}\right)$, we can describe the dynamics of heterotrophic morphs by the following Lotka-Volterra equations:

$$
\frac{d u_{i}}{d t}=-d_{i} u_{i}+\sum_{j=0}^{n} \lambda \frac{s_{j}}{s_{i}} a \gamma_{i j}\left(x_{i}, x_{j}\right) u_{j} u_{i}-\sum_{j=1}^{n} a \gamma_{j i}\left(x_{j}, x_{i}\right) u_{i} u_{j}
$$

where the intrinsic death rate $d_{i}=\exp \left(-q x_{i}\right)$, following Brännström et $\mathrm{al}^{18} ; a$ is the attack rate; and the consumption kernel $\gamma_{i j}$ describes the probability of a morph $i$ individual successfully hunting and consuming a morph $j$ individual after the encounter and is assumed to follow a normal distribution,

$$
\gamma_{i j}\left(x_{i}, x_{j}\right)=N\left(\mu, \sigma_{p}, x_{i}-x_{j}\right),
$$

where $\mu$ defines the optimal consumer to resource body size ratio at which the consumer can make the most successful attacks, and $\sigma_{p}$ describes the dietary niche width (ie, the standard deviation of the consumption kernel). Conversion parameter $\lambda$ is the fraction of captured resources that a consumer uses for its reproduction. The demographic dynamics of the autotrophic morph can be described as follows:

$$
\frac{d u_{0}}{d t}=r u_{0}\left(1-\frac{u_{0}}{k_{2}}\right)-\sum_{j=1}^{n} a \gamma_{j 0} u_{0} u_{j}
$$

where $r$ is the intrinsic growth rate of the autotrophic resource; $k_{2}$ is the carrying capacity such that $r / k_{2}$ depicts the strength of density dependence in the resource. Considering a mutant with trait $x^{\prime}$ appearing in a monomorphic resident population with trait $x_{1}$ at equilibrium $u_{1}{ }^{*}$ (also that the autotroph is at equilibrium $u_{0}{ }^{*}$ ), the invasion fitness is given by

$$
f_{1}\left(x_{1}, x^{\prime}\right)=-d\left(x^{\prime}\right)+\sum_{j=0}^{1} \lambda \frac{S_{j}}{S^{\prime}} a \gamma\left(x^{\prime}, x_{j}\right) u_{j}^{*}-a \gamma\left(x_{1}, x^{\prime}\right) u_{1}^{*}
$$

and the selection gradient on the heterotroph trait is given by

$$
g_{1}\left(x_{1}\right)=-\partial_{x^{\prime}} d\left(x_{1}\right)+\sum_{j=0}^{1} \lambda \underset{S}{S} a u_{j}^{*} \partial_{x^{\prime}} \gamma\left(x_{1}, x_{j}\right)-a u_{1}{ }^{*} \partial_{x^{\prime}} \gamma\left(x_{1}, x_{1}\right)
$$

A simulation based on the AD model was illustrated in Figure 5, clearly demonstrating the possibility of evolutionary branching driven by intertrophic interactions. The top predator (largest trait value) gradually increases its body size, while the body size gap between the top predator and the autotroph is gradually filled up by meso-predators 


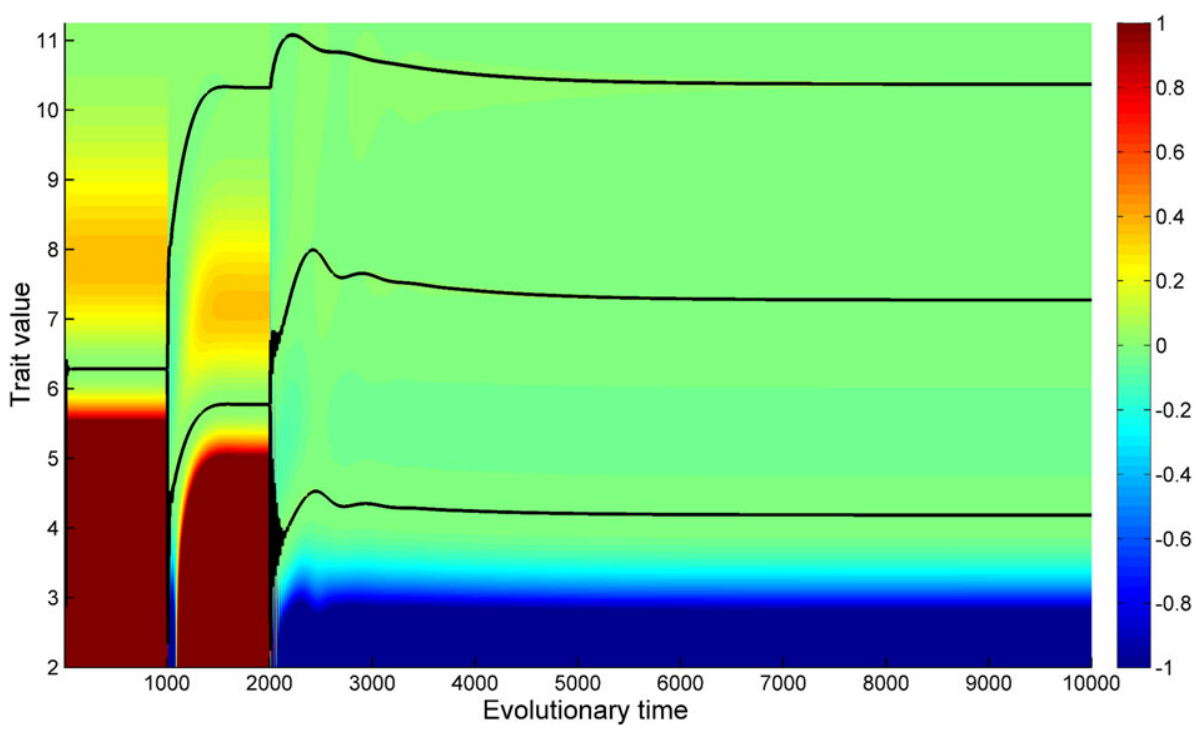

FIGURE 5 Trait diversification in a food web. Lines represent trait dynamics along the evolutionary time, starting from a monomorphic heterotroph population and diversifying into different morphs. Background colour represent the invasion fitness. Autotroph trait is constant setting at 1 . Parameter values are the same as in Brännström et $\mathrm{al}^{18}$ [Colour figure can be viewed at wileyonlinelibrary.com]

(Figure 5). The strength of disruptive selection, measured by the curvature of fitness landscape at the singularity, increases with the increase of dietary width $\left(\sigma_{p}\right)^{5,18}$ This suggests that diversification, at least the first evolutionary branching, is easier in communities with more generalists than specialists. Importantly, parameters that foster the first evolutionary branching are not necessarily suitable for biodiversity maintenance. ${ }^{18}$ Although many laboratory experiments have been designed to determine factors that favour the initial diversification, ${ }^{72-74}$ more research is needed to understand how diversity is maintained along the evolutionary trajectory (see also Dercole ${ }^{68}$ and Kisdi et al ${ }^{67}$ where an evolutionary suicide follows branching). Other factors may play an increasingly critical role for biodiversity maintenance with the increase of species richness but have only trivial effects when the system is species poor. ${ }^{5}$ Moreover, with the increase of dietary width, the body-size ratio between adjacent morphs declines, and the food chains become longer as the mean predator-prey body size ratios declines. ${ }^{75}$ Since there is a strong correlation between body size and trophic level, ${ }^{71}$ a generalist top predator often has a larger body size than a specialist.

\section{7 | CONCLUSIONS}

Coevolution is a major source of adaptive diversification. Mutualistic and antagonistic interactions between species can strongly affect each other's fitness and trigger density-dependent selection that is essential for both evolutionary branching and diversity maintenance. ${ }^{10,76}$ As a species often has multiple functions in a community, eg, as prey, predator, and pollinator, whether a specific biotic interaction drives adaptive diversification is often context based. ${ }^{46}$ Resource competition has been shown to trigger niche-filling diversification, with a narrower competition kernel supporting easier diversification and higher species richness. Intratrophic competition plays the same role in mutualistic and antagonistic coevolution, with narrower competition kernel (weaker trait specific competition) more easily triggering disruptive selection and evolutionary branching.

Bipartite networks are common in ecology, with involved species naturally divided into 2 functional groups. In mutualistic systems, adaptive diversification only happens to the group with a narrower competition kernel, indicating stronger negative frequency dependence. ${ }^{10,50}$ Low tolerance to cross-trophic trait difference $\left(\sigma_{p}\right)$ leads to matched traits but could then lead to diversification when competition is relatively strong. High tolerance as in many generalists often leads to bias between interacting traits. Strong cross-trophic interactions often lead to convergence evolution towards an ESS, while species involving weak cross-trophic interactions behave independently as resource competition within its own functional group. ${ }^{77}$ Mutualistic interactions can trigger diversification when the cross-trophic interaction is moderate so that asymmetric fitness between the 2 groups often triggers the diversification in the less fit group. ${ }^{78}$ The 2 functional groups in antagonistic systems are not symmetrical as in mutualistic systems. Consequently, generalist predators (not 
prey specific) are more susceptible to disruptive selection and diversification, while the diversification of specialist predators follows the branching in the prey, ${ }^{51}$ although both intercompetition and intracompetition within each group can override these claims by affecting adaptive diversification. Food webs, a more generic antagonistic system than the bipartite network, behave rather similarly. Disruptive selection is strengthened when species are dietary generalists, and wider diets also support top predators with larger body size. Of course, factors for initial diversification may be different from those that influence eventual diversity maintenance, similar to the case of community succession where pioneer species often have distinct traits from climax species at later succession stages.

Coevolutionary networks provide an ideal model of complex adaptive systems. ${ }^{5}$ In this system, it is important to choose adaptively with whom to interact (habitat and diet selection) or to avoid (antipredation strategies). ${ }^{79,80}$ Such interactions are often assortative as modelled by the function of $\alpha$ and $\gamma$ used in above models. Assortative mating is important for evolutionary branching in sexual populations, while assortative cross-trophic interactions are essential for adaptive diversification in coevolutionary systems. Such preferential interactions could simply arise from optimal or adaptive foraging where species aim to maximise their energy intake rate, ${ }^{81}$ while being undermined by others during their maximisation. This is a grand multiplayer game. To survive in such a game, species often have to have multiple contingency plans with which to handle ecological or evolutionary selection pressures. Ecologically, for survival, species can adjust their distributions via fitness-dependent dispersal ${ }^{82}$ and partition spatiotemporal niches, ${ }^{83}$ forming complex assemblage patterns. ${ }^{84}$ For reducing evolutionary pressures, species can modify their functional and morphological traits ${ }^{85}$ under the constraints of functional trade-offs. ${ }^{86}$ Adaptive dynamics has been proven to be a powerful tool for exploring the rich possibility of ecological dynamics ${ }^{87,88}$ and evolutionary trajectories ${ }^{5,10,18,78}$ in multispecies ecological networks. Adaptive diversification from coevolution can be considered a possible pathway leading to contemporary multispecies ecological networks.

\section{ACKNOWLEDGEMENTS}

This work was presented as a plenary lecture and 2 associated presentations at the BioMath 2017 Meeting, held in the Kruger National Park, South Africa. We are grateful to the following colleagues for inspiration discussions on the topic: Ulf Dieckmann, Åke Brännström, Feng Zhang, Anton Pauw, Jaco Le Roux, John Terblanche, Fabio Dercole, Fabio Della Rossa, and Sergio Rinaldi, as well as the many interesting discussions during the meeting. $\mathrm{CH}$ is supported by the National Research Foundation of South Africa (grants 89967 and 109244) and the South African Research Chair Initiative. PL acknowledges support from the National Research Foundation of South Africa. HOM acknowledges the postdoctoral fellowship from the DST-NRF Centre of Excellence in Mathematical and Statistical Sciences (CoE-MaSS; grant BA2017/136).

\section{ORCID}

Cang Hui (D) http://orcid.org/0000-0002-3660-8160

\section{REFERENCES}

1. Wright S. Surfaces of selective value revisited. Am Nat. 1988;131:115-123.

2. Ridley M. Evolution. Oxford: Blackwell; 2004.

3. Allen JA, Clarke BC. Frequency-dependent selection - homage to Poulton, E.B. Biol J Linn Soc. 1984;23:15-18.

4. Dercole F, Ferrière R, Rinaldi S. Ecological bistability and evolutionary reversals under asymmetrical competition. Evolution. 2002;56:1081-1090.

5. Hui C, Minoarivelo HO, Nuwagaba S, Ramanantoanina A. Adaptive diversification in coevolutionary systems. In: Pontarotti P, ed. Evolutionary Biology: Biodiversification from Genotype to Phenotype. Springer: Switzerland; 2015:167-186.

6. Darwin C. On the Various Contrivances by Which British and Foreign Orchids are Fertilised by Insects, and on the Good Effects of Intercrossing. London: John Murray; 1862.

7. Lengyel S, Gove AD, Latimer AM, Majer JD, Dunn RR. Ants sow the seeds of global diversification in flowering plants. PLoS One. 2009;4: e5480

8. Becerra JX, Venable DL. Macroevolution of insect-plant associations: the relevance of host biogeography to host affiliation. Proc Natl Acad Sci U S A. 1999;96:12626-12631. 
9. Moran NA, Baumann P. Phylogenetics of cytoplasmically inherited microorganisms of arthropods. Trends Ecol Evol. 1994;9:15-20.

10. Doebeli M, Ispolatov I. Complexity and diversity. Science. 2010;328:494-497.

11. Ehrlich PR, Raven PH. Butterflies and plants: a study in coevolution. Evolution. 1964;18:586-608.

12. Rezende EL, Lavabre JE, Guimarães PR, Jordano P, Bascompte J. Non-random coextinctions in phylogenetically structured mutualistic networks. Nature. 2007;448:925-928.

13. Rezende EL, Jordano P, Bascompte J. Effects of phenotypic complementarity and phylogeny on the nested structure of mutualistic networks. Oikos. 2007;116:1919-1929.

14. Guimarães PR, Jordano P, Thompson JN. Evolution and coevolution in mutualistic networks. Ecol Lett. 2011;14:877-885.

15. Nuismer SL, Gomeulkiewiez R, Ridenhour BJ. When is correlation coevolution? Am Nat. 2010;175:525-537.

16. Minoarivelo HO, Hui C, Terblanche JS, Kosakovsky Pond SL, Scheffler K. Detecting phylogenetic signal in mutualistic interaction networks using a Markov process model. Oikos. 2014;123:1250-1260.

17. Doebeli M, Dieckmann U. Evolutionary branching and sympatric speciation caused by different types of ecological interactions. Am Nat. 2000;156:S77-S101.

18. Brännström ̊̊, Loeuille N, Loreau M, Dieckmann U. Emergence and maintenance of biodiversity in an evolutionary food-web model. Theor Ecol. 2011;4:467-478.

19. Dieckmann U, Law R. The dynamical theory of coevolution: a derivative from stochastic ecological processes. J Math Biol. 1996;34:579-612.

20. Geritz SAH, Kisdi É, Meszéna G, Metz JAJ. Evolutionary singular strategies and the adaptive growth and branching of the evolutionary tree. Evol Ecol. 1998;12:35-57.

21. Dercole F, Rinaldi S. Analysis of Evolutionary Processes: The Adaptive Dynamics Approach and Its Applications. Princeton: Princeton University Press; 2008.

22. Parvinen K. Evolution of migration in a metapopulation. Bull Math Biol. 1999;61:531-550.

23. Parvinen K. Evolutionary branching of dispersal strategies in structured metapopulations. J Math Biol. 2002;45:106-124.

24. Dercole F, Loiacono D, Rinaldi S. Synchronization in population networks: a byproduct of Darwinian evolution? Int J Bifurcation Chaos. 2007;7:2435-2446.

25. Gavrilets S. Fitness Landscapes and the Origin of Species. Princeton: Princeton University Press; 2004.

26. Otta SP, Day T. A Biologist's Guide to Mathematical Modelling in Ecology and Evolution. Princeton: Princeton University Press; 2007.

27. Dercole F, Geritz SAH. Unfolding the resident-invader dynamics of similar strategies. J Theor Biol. 2016;394:231-254.

28. Hui C, Richardson DM. Invasion Dynamics. Oxford: Oxford University Press; 2017.

29. Hui C, Richardson DM, Landi P, Minoarivelo HO, Garnas J, Roy HE. Defining invasiveness and invasibility in ecological networks. Biol Invasions. 2016;18:971-983.

30. Nowak M, Sigmund K. The evolution of stochastic strategies in the prisoner's dilemma. Acta Appl Math. 1990;20:247-265.

31. Abrams PA, Harada Y, Matsuda H. On the relationship between quantitative genetic and ESS models. Evolution. 1993;47:982-985.

32. Metz JAJ, Nisbet R, Geritz SAH. How should we define 'fitness' for general ecological scenarios? Trends Ecol Evol. 1992;7:198-202.

33. Waxman D, Gavrilets S. 20 questions on adaptive dynamics. J Evol Biol. 2005;18:1139-1154.

34. Della Rossa F, Dercole F, Landi P. The branching bifurcation of adaptive dynamics. Int J Bifurcation Chaos. 2015;25: 1540001

35. Dercole F, Della Rossa F, Landi P. The transition from evolutionary stability to branching: a catastrophic evolutionary shift. Sci Rep. 2016;6: 26310

36. Dercole F. The ecology of asexual pairwise interactions: the generalized law of mass action. Theor Ecol. 2016;9:299-321.

37. Gyllenberg M, Parvinen K, Dieckmann U. Evolutionary suicide and evolution of dispersal in structured populations. $J$ Math Biol. 2002;45:79-105.

38. Zhang F, Hui C, Pauw A. Adaptive divergence in Darwin's race: how coevolution can generate trait diversity in a pollination system. Evolution. 2013;67:548-560.

39. Birand A, Barany E. Evolutionary dynamics through multispecies competition. Theor Ecol. 2014;7:367-379.

40. Dercole F, Dieckmann U, Obersteiner M, Rinaldi S. Adaptive dynamics and technological change. Technovation. 2008;28:335-348.

41. Godsoe W, Yoder JB, Smith CI, Pellmyr O. Coevolution and divergence in the Joshua tree/yucca moth mutualism. Am Nat. 2008;171:816823.

42. Le Roux JJ, Hui C, Keet J-H, Ellis AG. Co-introduction vs ecological fitting as pathways to the establishment of effective mutualisms during biological invasions. New Phytol. 2017;215:1354-1360.

43. Pellmyr O, Leebens-Mack J. Forty million years of mutualism: evidence for Eocene origin of the yucca-yucca moth association. Proc Natl Acad Sci U S A. 1999;96:9178-9183. 
44. Gómez JM, Verdú M. Mutualism with plants drives primate diversification. Syst Biol. 2012;61:567-577.

45. Weber MG, Agrawal AA. Defense mutualisms enhance plant diversification. Proc Natl Acad Sci U S A. 2014;111:16442-16447.

46. Raimundo RL, Gilbert JP, Hembry DH, Guimarães PR. Conflicting selection in the course of adaptive diversification: the interplay between mutualism and intraspecific competition. Am Nat. 2014;183:363-375.

47. Ferrière R, Bronstein JL, Rinaldi S, Law R, Gauduchon M. Cheating and the evolutionary stability of mutualisms. Proc R Soc Lond B Biol Sci. 2002;269:773-780.

48. Dercole F. Border collision bifurcations in the evolution of mutualistic interactions. Int J Bifurcation Chaos. 2005;15:2179-2190.

49. Bürger R, Schneider KA, Willensdorfer M. The conditions for speciation through intraspecific competition. Evolution. 2006;60:2185-2206.

50. Day T, Young KA. Competitive and facilitative evolutionary diversification. Bioscience. 2004;54:101-109.

51. Landi P, Dercole F, Rinaldi S. Branching scenarios in eco-evolutionary prey-predator models. SIAM J Appl Math. 2013;73:1634-1658.

52. Landi P, Hui C, Dieckmann U. Fisheries-induced disruptive selection. J Theor Biol. 2015;365:204-216.

53. Hanifin CT, Brodie ED Jr, Brodie ED III. Phenotypic mismatches reveal escape from arms-race coevolution. PLoS Biol. 2008;6:471-481.

54. Fraenkel G. The raison d'être of secondary plant substances. Science. 1959;129:1466-1470.

55. Broeckhoven C, Diedericks G, Hui C, Makhubo BG, Mouton PLFN. Enemy at the gates: rapid defensive trait diversification in an adaptive radiation of lizards. Evolution. 2016;70:2647-2656.

56. Mallet J. Shift happens! Shift balance and the evolution of diversity in warning color and mimicry. Ecol Entomol. 2010;35:90-104.

57. Rothstein SI, Robinson SK. Parasitic Birds and Their Hosts: Studies in Coevolution. New York: Oxford University Press; 1998.

58. Decaestecker E, Gaba S, Raeymaekers JAM, et al. Host-parasite 'Red Queen’ dynamics archived in pond sediment. Nature. 2007;450:870873.

59. Payne R, Muenchhoff M, Mann J, et al. Impact of HLA-driven HIV adaptation on virulence in populations of high HIV seroprevalence. Proc Natl Acad Sci USA. 2014;111:E5393-E5400.

60. Marston MF, Pierciey FJ, Shepard A, et al. Rapid diversification of coevolving marine synechococcus and a virus. Proc Natl Acad Sci USA. 2012;109:4544-4549.

61. Landi P, Dercole F. The social diversification of fashion. J Math Sociol. 2016;40:185-205.

62. Van Valen L. A new evolutionary law. Evolutionary Theory. 1973;1:1-30.

63. Dieckmann U, Marrow U, Law R. Evolutionary cycling in predator-prey interactions: population dynamics and the Red Queen. $J$ Theor Biol. 1995;176:91-102.

64. Dercole F, Gragnani A, Ferrière R, Rinaldi S. Coevolution of slow-fast populations: an application to prey-predator systems. Proc $R$ Soc Lond B Biol Sci. 2006;273:983-990.

65. Dercole F, Ferrière R, Rinaldi S. Chaotic Red Queen coevolution in three-species food chains. Proc R Soc Lond B Biol Sci. 2010;277:2321-2330.

66. Dercole F, Rinaldi S. Evolutionary dynamics can be chaotic: a first example. Int J Bifurcation Chaos. 2010;20:3473-3485.

67. Kisdi É, Jacobs FJA, Geritz SAH. Red Queen evolution by cycles of evolutionary branching and extinction. Selection. 2001;2:161-176.

68. Dercole F. Remarks on branching extinction evolutionary cycles. J Math Biol. 2003;47:569-580.

69. Dercole F, Rinaldi S. Evolution of cannibalism: scenarios derived from adaptive dynamics. Theor Popul Biol. 2002;62:365-374.

70. Cattin M-F, Bersier L-F, Banašek-Richter C, Baltensperger R, Gabriel J-P. Phylogenetic constraints and adaptation explain food-web structure. Nature. 2004;427:835-838.

71. Loeuille N, Loreau M. Evolutionary emergence size-structured food webs. Proc Natl Acad Sci USA. 2005;102:5761-5766.

72. Buckling A, Rainey PB. The role of parasites on sympatric and allopatric diversification of hosts. Nature. 2002;420:496-499.

73. Friesen ML, Saxer G, Travisano M, Doebeli M. Experimental evidence for sympatric ecological diversification due to frequency-dependent competition in Escherichia Coli. Evolution. 2004;58:245-260.

74. Nosil P, Crespi BJ. Experimental evidence that predation promotes divergence in adaptive radiation. Proc Natl Acad Sci U S A. 2006;103: S9090-S9095.

75. Jennings S, Warr KJ. Smaller predator-prey body size ratios in longer food chains. Proc R Soc Lond B Biol Sci. 2003;270:1413-1417.

76. Biktashev VN. A simple mathematical model of gradual Darwinian evolution: emergence of a Gaussian trait distribution in adaptation along a fitness gradient. J Math Biol. 2014;68:1225-1248.

77. Minoarivelo HO, Hui C. Invading a mutualistic network: to be or not to be similar. Ecol Evol. 2016;6:4981-4996.

78. Minoarivelo HO, Hui C. Trait-mediated interaction leads to structural emergence in mutualistic networks. Evolutionary Ecology. 2016;30:105-121.

79. Zhang F, Hui C, Terblanche JS. An interaction switch predicts the nested architecture of mutualistic networks. Ecol Lett. 2011;14:797-803. 
80. Nuwagaba S, Zhang F, Hui C. A hybrid behavioural rule of adaptation and drift explains the emergent architecture of antagonistic networks. Proc R Soc Lond B Biol Sci. 2015;282: 20150320.

81. Zhang F, Hui C. Recent experience-driven behaviour optimizes foraging. Anim Behav. 2014;88:13-19.

82. Hui C, Roura-Pascual N, Brotons L, Robinson RA, Evans KL. Flexible dispersal strategies in native and non-native ranges: environmental quality and the 'good-stay, bad-disperse' rule. Ecography. 2012;35:1024-1032.

83. Hui C, Fox GA, Gurevitch J. Scaling up demography: consequences for population volatility and growth from landscape portfolios. Submitted.

84. Hui C, McGeoch MA. Zeta diversity as a concept and metric that unifies incidence-based biodiversity patterns. Am Nat. 2014;184:684-694.

85. Berthouly-Salazar C, Hui C, Blackburn TM, et al. Long-distance dispersal maximizes evolutionary potential during rapid geographic range expansion. Mol Ecol. 2013;22:5793-5804.

86. Broeckhoven C, du Plessis A, Hui C. Functional trade-off between strength and thermal capacity of dermal armor: insights from girdled lizards. J Mech Behav Biomed Mater. 2017; in press. DOI:https://doi.org/10.1016/j.jmbbm.2017.06.007

87. Wilsenach J, Landi P, Hui C. Evolutionary fields can explain patterns of high-dimensional complexity in ecology. Physical Review E. 2017;95: 042401

88. Brännström Å, Johansson J, Loeuille N, et al. Modelling the ecology and evolution of communities: a review of past achievements, current efforts, and future promises. Evol Ecol Res. 2012;14:601-625.

How to cite this article: Hui C, Minoarivelo HO, Landi P. Modelling coevolution in ecological networks with adaptive dynamics. Math Meth Appl Sci. 2018;41:8407-8422. https://doi.org/10.1002/mma.4612 